\title{
State Estimation Using Virtual Measurement Information
}

\author{
Benjamin Noack and Uwe D. Hanebeck, \\ Intelligent Sensor-Actuator-Systems Laboratory (ISAS) \\ Institute for Anthropomatics and Robotics \\ Karlsruhe Institute of Technology (KIT), Germany \\ benjamin.noack@ieee.org, uwe.hanebeck@ieee.org
}

\begin{abstract}
The computation of an estimate for the unknown state of a dynamical system is a central challenge in many disciplines and applications. In general, the estimation quality is directly tied to the amount of sensor data available to the state estimation system. However, insights from virtual or missing observations may also convey exploitable information on the system's state. Such virtual measurement information may relate to constraints to which the state is subject. For instance, constraints to acceleration and turn rate of a mobile robot may apply and can be exploited. Analogously, missing observations that are attributable to obstacles can be translated into usable information, which is often referred to as negative sensor evidence. Such implicit information has to be reformulated into virtual measurement data in order to take advantage of it. As the Kalman filter and its derivatives are most widely used in state estimation applications, specific measurement and noise models for virtual observations are to be derived that can easily be integrated into the prediction-correction cycle of the Kalman filter. In this work, a set-membership representation of virtual measurement information is discussed.
\end{abstract}

Keywords: State Estimation, Kalman Filter, Virtual Measurements, Constraints, Negative Information

\section{Introduction}

State estimation has become a widely studied and applied research discipline and refers to the task of deriving an estimate of an unknown state from noisy and erroneous sensor data. In many applications, also virtual or even missing observations may convey information on the system's state that can be exploited. The core question in this regard is how to identify and model virtual sensor evidence.

In order to translate virtual sensor evidence into exploitable information, additional knowledge about the studied phenomenon, its environment, or infrastructure can be utilized. Typical types of virtual sensor evidence are:

State constraints directly apply to the process dynamics of the considered system. For instance, kinematic constraints on velocity and acceleration can be exploited to enhance the performance of target tracking systems [1]. For industrial process monitoring, constraints on the heat transfer can be used in order to improve estimation quality [2].

Environmental constraints can relate to a map that provides prior information about geographical constraints. An important example in target tracking is a road map [3], which can be modeled as a constraint on the target's position.
Missing observations can, for instance, be exploited in robot mapping if prior knowledge about the probability of detecting a landmark is available. In this case, landmarks being not in sensor range also contribute to estimating the robot's position [4]. In event-based state estimation, sensor readings are typically transmitted when a certain threshold is exceeded. Between events, virtual measurements can be derived from the event-triggering criterion [5]. Another example is a ground moving target indicator radar [6, 7], where missing observations imply that targets do not reach the minimum detectable velocity, i.e., they can be viewed as indirect velocity measurements.

The concept of virtual measurements requires special care and involves considerable difficulties concerning the design of appropriate models as well as the interpretation of missing observations. For instance, the estimation system cannot discern whether the state to be monitored is out of range or the sensors simply fail to detect it. The use of an adequate model is crucial but typically raises the complexity of the filtering method. In particular, equality constraints on the state have been widely studied as they can easily be integrated into the Kalman filter algorithm. These constraints can be modeled as 
error-free pseudo measurements of state components, and the Kalman filter can be extended by an according update step. However, equality constraints are often difficult to justify in practice and can be too restrictive. By contrast, inequality constraints are practically more relevant but may lead to complicated optimization problems. Similarly, models for missing observations often require the design of non-Gaussian likelihoods and lead to nonlinear estimation problems. In many applications, implicit information can rather be associated with a set-membership representation than with a stochastic characterization. For instance, an obstacle that prevents a mobile object to be detected is related an occluded area, which may serve as negative sensor evidence, or the detection threshold of a ground moving target indicator translates to a set of velocity values. Accordingly, constraints such as a road can be modeled with a set in order to take their width or extend into account. Such set-membership information can easily be integrated into the Kalman filtering scheme when being modeled as ellipsoidal set. In particular, the application to ellipsoidal constraints on the state and the exploitation of negative sensor evidence are discussed in this work.

\section{Notations}

The following notations are used. Real-valued vectors are denoted by underlined variables $\underline{x}$, and boldface, lowercase letters $\underline{x}$ represent random quantities. Matrices $\mathbf{C} \in \mathbb{R}^{n \times n}$ are written in uppercase boldface letters. The matrices $\mathbf{C}^{-1}$ and $\mathbf{C}^{\mathrm{T}}$ are the inverse and transpose, respectively. The vector $\underline{\hat{x}}$ is used for the mean of a random variable, an estimate of an uncertain quantity, or an observation. The matrix $\mathbf{I}$ is the identity matrix of appropriate dimension. By $\mathcal{N}(\underline{\hat{x}}, \mathbf{C})$, the normal distribution with mean $\underline{\hat{x}}$ and covariance matrix $\mathbf{C}$ is denoted. An ellipsoid with center $\underline{c}$ and shape matrix $\mathbf{X}$ is defined by $\mathcal{E}(\underline{c}, \mathbf{X})=$ $\left\{\underline{x} \in \mathbb{R}^{n} \mid(\underline{c}-\underline{x})^{\mathrm{T}} \mathbf{X}^{-1}(\underline{c}-\underline{x}) \leq 1\right\}$. An element of $\mathcal{E}(\underline{c}, \mathbf{X})$ is denoted by $\underline{\mathbb{Q}}$.

\section{Physical and Virtual Measurements}

In state estimation theory, uncertain quantities are typically characterized by random variables. As such, measurement uncertainty is commonly modeled as an additive white noise term $\underline{\mathbf{v}}_{k}$ affecting an observation

$$
\underline{\mathbf{z}}_{k}=\mathbf{H}_{k} \underline{\mathbf{x}}_{k}+\underline{\mathbf{v}}_{k}
$$

where $\underline{x}_{k} \in \mathbb{R}^{n_{x}}$ is the state to be estimated and $\mathbf{H}_{k} \in \mathbb{R}^{n_{z} \times n_{x}}$ is the measurement matrix at the discrete time step $k$. The statistics of $\underline{\mathbf{v}}_{k}$ are defined by the error covariance matrix $\mathbf{C}_{k}^{\mathbf{v}}=\operatorname{Cov}\left(\underline{\mathbf{v}}_{k}\right) \in \mathbb{R}^{n_{z} \times n_{z}}$. Such a probabilistic representation is generally employed to model the measurement uncertainty involved in physical sensor readings.

In many situations, further knowledge about the system state can be exploited. Against the background of target tracking applications, several examples have been discussed in [7]. In group tracking, specific models can be derived that take into account unresolved targets in a group of closely spaced targets. Accordingly, mutual occlusions among multiple extended targets can be represented by an occlusion likelihood [8] so that the position of occluded targets can still be updated. For this type of virtual measurement information, the term negative information has been established [9, 10]. Negative sensor evidence is based on prior knowledge about the likelihood of not observing the state or components of the state. The named examples utilize probabilistic characterizations of negative information which implies that a stochastic model such as (1) is applied. However, the definition and computation of the measurement likelihoods is a common problem.

An alternative direction is a set-membership approach to modeling negative information. The intuition behind this approach is that constraints or occlusions often refer to bounded regions, to which the system's state belongs. For instance, occluded targets are located in the area behind an obstacle. Similarly, the extend of a road may serve as a constraint on the position of wheeled vehicles. In order to attain a simple estimation scheme in the subsequent section, ellipsoidal sets are employed to model virtual information, and the virtual measurement equation is defined by

$$
\mathbf{H}_{k}^{*} \underline{\mathbf{x}}_{k} \in \mathcal{E}\left(\underline{z}_{k}^{*}, \mathbf{X}_{k}^{*}\right)
$$

where $\underline{z}_{k}^{*}$ and $\mathbf{X}_{k}^{*}$ are the center and the shape matrix of the ellipsoid,respectively. By exploiting the symmetry of the ellipsoid, the measurement equation can be reformulated to

$$
\underline{z}_{k}^{*}=\mathbf{H}_{k}^{*} \underline{\mathbf{x}}_{k}+\underline{\mathbb{e}}_{k}
$$

with the set-membership term $\underline{e}_{k} \in \mathcal{E}\left(\underline{0}, \mathbf{X}_{k}^{*}\right)$. This representation bears a strong resemblance with (1), and the term $\underline{e}_{k}$ has the same parameterization as $\underline{\mathbf{v}}_{k}$. It must be noticed that an ellipsoidal representation of uncertainty does neither correspond to a Gaussian distribution nor a uniform distribution. It is one major advantage of set-membership representations that no probabilities need to be assigned, which can significantly ease the modeling of virtual informa- 
tion. The particular advantage of ellipsoidal representations is that the model (2) for virtual measurement information can easily be integrated into the widely used Kalman filtering scheme.

\section{Kalman Filtering with Physical and Virtual Measurements}

The standard Kalman filter computes an estimate $\underline{\hat{x}}_{k}^{\mathrm{e}}$ that is designed to minimize the mean squared error, which complies with minimizing the trace of the error covariance matrix $\mathbf{C}_{k}^{\mathrm{e}}=$ $\operatorname{Cov}\left(\underline{\tilde{x}}_{k}\right)$ with

$$
\underline{\tilde{\mathbf{x}}}_{k}=\underline{\hat{x}}_{k}^{\mathrm{e}}-\underline{\mathbf{x}}_{k}
$$

For the purpose of incorporating set-membership measurement information, the Kalman filter is generalized based on the concepts in [11] and [12]. Due to the ellipsoidal representation (2) of virtual measurements, the estimation error (3) is not only composed of a random variable $\underline{\tilde{x}}_{k}$ but also comprises a setmembership component $\underline{\underline{x}}_{k}$, i.e.,

$$
\underline{\tilde{\mathbf{x}}}_{k}+\underline{\underline{x}}_{k}=\underline{\hat{x}}_{k}^{\mathrm{e}}-\underline{\mathbf{x}}_{k} .
$$

The error of an estimate $\underline{\hat{x}}_{k}^{\mathrm{e}}$ is therefore characterized by a covariance matrix $\mathbf{C}_{k}^{\mathrm{e}}=\operatorname{Cov}\left(\underline{\tilde{x}}_{k}\right)$ and an ellipsoidal shape matrix $\mathbf{X}_{k}^{\mathrm{e}}$ with $\underline{x}_{k} \in$ $\mathcal{E}\left(\underline{0}, \mathbf{X}_{k}^{\mathrm{e}}\right)$. The objective is then to minimize the squared error

$$
\begin{aligned}
& \mathrm{E}\left[\left\|\underline{\hat{x}}_{k}^{\mathrm{e}}-\underline{\mathbf{x}}_{k}\right\|_{2}^{2}\right]=\mathrm{E}\left[\left(\underline{\hat{x}}_{k}^{\mathrm{e}}-\underline{\mathbf{x}}_{k}\right)^{\mathrm{T}}\left(\underline{\hat{x}}_{k}^{\mathrm{e}}-\underline{\mathbf{x}}_{k}\right)\right] \\
& =\mathrm{E}\left[\left(\underline{\tilde{\mathbf{x}}}_{k}\right)^{\mathrm{T}}\left(\underline{\tilde{\mathbf{x}}}_{k}\right)\right]+\left(\underline{\underline{x}}_{k}\right)^{\mathrm{T}}\left(\underline{\underline{x}}_{k}\right) \\
& \leq \operatorname{trace}\left(\mathbf{C}_{k}^{\mathrm{e}}\right)+\operatorname{trace}\left(\mathbf{X}_{k}^{\mathrm{e}}\right),
\end{aligned}
$$

with $\mathrm{E}\left[\left(\underline{\tilde{x}}_{k}\right)^{\mathrm{T}}\left(\underline{\tilde{x}}_{k}\right)\right]=\operatorname{trace}\left(\mathbf{C}_{k}^{\mathrm{e}}\right)$ and $\left(\underline{\underline{x}}_{k}\right)^{\mathrm{T}}\left(\underline{\underline{x}}_{k}\right) \leq$ $\operatorname{trace}\left(\mathbf{X}_{k}^{\mathrm{e}}\right)$. The latter inequality holds for every element in the ellipsoid $\mathcal{E}\left(\underline{0}, \mathbf{X}_{k}^{\mathrm{e}}\right)$. Additionally, the errors $\underline{\tilde{x}}_{k}$ and $\underline{x}_{k}$ are assumed to be uncorrelated. In a first step, the generalization of the Kalman filter is reviewed. In the second step, its application to virtual measurement information is discussed.

\subsection{Generalized Kalman Filter}

The generalized Kalman filter [12] is designed for process and sensor models that are simultaneously affected by stochastic and setmembership uncertainties. In particular, sensor models in the form of

$$
\underline{\mathbf{z}}_{k}=\mathbf{H}_{k} \underline{\mathbf{x}}_{k}+\underline{\mathbf{v}}_{k}+\underline{\mathbb{e}}_{k}
$$

with $\underline{\mathbf{v}}_{k} \sim \mathcal{N}\left(\underline{0}, \mathbf{C}_{k}^{\mathbf{v}}\right)$ and $\underline{\mathbb{e}}_{k} \in \mathcal{E}\left(\underline{0}, \mathbf{X}_{k}^{v}\right)$ are considered. Accordingly, the process model has the form

$$
\underline{\mathbf{x}}_{k+1}=\mathbf{A}_{k} \underline{\mathbf{x}}_{k}+\mathbf{B}_{k} \underline{u}_{k}+\underline{\mathbf{w}}_{k}+\underline{\mathbb{d}}_{k}
$$

with the system matrix $\mathbf{A}_{k} \in \mathbb{R}^{n_{x} \times n_{x}}$ and process noise terms $\underline{\mathbf{w}}_{k} \sim \mathcal{N}\left(\underline{0}, \mathbf{C}_{k}^{\mathbf{w}}\right)$ and $\underline{\mathbb{d}}_{k} \in$ $\mathcal{E}\left(\underline{0}, \mathbf{X}_{k}^{\mathbf{w}}\right)$. The vector $\underline{u}_{k} \in \mathbb{R}^{n_{u}}$ is a possible control input with control matrix $\mathbf{B}_{k} \in \mathbb{R}^{n_{x} \times n_{u}}$. The measurement update and the prediction step are carried out by means of the following recursive formulas. The parameters are initialized with the prior estimate $\underline{\hat{x}}_{0}^{\mathrm{p}}$ and error matrices $\mathbf{C}_{0}^{\mathrm{p}}$ and $\mathbf{X}_{0}^{\mathrm{p}}$.

Update Step: For the filtering step, the gain is computed to account for both stochastic and set-membership measurement uncertainties in sensor data and yields

$$
\begin{aligned}
& \mathbf{K}_{k}(\omega)=\left(\frac{1}{\omega} \mathbf{X}_{k}^{\mathrm{p}} \mathbf{H}_{k}^{\mathrm{T}}+\mathbf{C}_{k}^{\mathrm{p}} \mathbf{H}_{k}^{\mathrm{T}}\right) . \\
& \left(\frac{1}{\omega} \mathbf{H}_{k} \mathbf{X}_{k}^{\mathrm{p}} \mathbf{H}_{k}^{\mathrm{T}}+\frac{1}{1-\omega} \mathbf{X}_{k}^{\vee}+\mathbf{H}_{k} \mathbf{C}_{k}^{\mathrm{p}} \mathbf{H}^{\mathrm{T}}+\mathbf{C}_{k}^{\mathbf{v}}\right)^{-1},
\end{aligned}
$$

which can be derived by minimizing the righthand side of the inequality (4). Evidently, the gain also depends on the weighting parameter $\omega \in(0,1)$. Estimate and covariance matrix are updated according to

$$
\underline{\hat{x}}_{k}^{\mathrm{e}}(\omega)=\left(\mathbf{I}-\mathbf{K}_{k}(\omega) \mathbf{H}_{k}\right) \underline{\hat{x}}_{k}^{\mathrm{p}}+\mathbf{K}_{k}(\omega) \underline{\hat{z}}_{k},
$$

and

$$
\begin{aligned}
\mathbf{C}_{k}^{\mathrm{e}}(\omega)= & \left(\mathbf{I}-\mathbf{K}_{k}(\omega) \mathbf{H}_{k}\right) \mathbf{C}_{k}^{\mathrm{p}}\left(\mathbf{I}-\mathbf{K}_{k}(\omega) \mathbf{H}_{k}\right)^{\mathrm{T}} \\
& +\mathbf{K}_{k}(\omega) \mathbf{C}_{k}^{\mathrm{v}} \mathbf{K}_{k}(\omega)^{\mathrm{T}},
\end{aligned}
$$

respectively. These formulas correspond to the standard Kalman filter if set-membership errors are absent, i.e, $\mathbf{X}_{k}^{\mathrm{p}}=\mathbf{0}$ and $\mathbf{X}_{k}^{\vee}=\mathbf{0}$. In this case, the matrix (7) reduces to the standard Kalman gain. In the presence of setmembership uncertainties, the shape matrix has to be updated according to

$$
\begin{aligned}
\mathbf{X}_{k}^{\mathrm{e}}(\omega)= & \frac{1}{\omega}\left(\mathbf{I}-\mathbf{K}_{k}(\omega) \mathbf{H}_{k}\right) \mathbf{X}_{k}^{\mathrm{p}}\left(\mathbf{I}-\mathbf{K}_{k}(\omega) \mathbf{H}_{k}\right)^{\mathrm{T}} \\
& +\frac{1}{1-\omega} \mathbf{K}_{k}(\omega) \mathbf{X}_{k}^{\vee} \mathbf{K}_{k}(\omega)^{\mathrm{T}}
\end{aligned}
$$

which is the shape matrix of an enclosing ellipsoid for the set-membership estimation error. More precisely, $\mathcal{E}\left(\underline{0}, \mathbf{X}_{k}^{\mathrm{e}}\right)$ is an outer ellipsoidal bound of the Minkowski sum $\left(\mathbf{I}-\mathbf{K}_{k}(\omega) \mathbf{H}_{k}\right) \mathcal{E}\left(\underline{\hat{x}}_{k}^{\mathrm{p}}, \mathbf{X}_{k}^{\mathrm{p}}\right) \oplus \mathbf{K}_{k} \mathcal{E}\left(\underline{0}, \mathbf{X}_{k}^{\vee}\right)$. Each $\omega \in(0,1)$ is admissible, but the parameter is typically determined to minimize trace $\left(\mathbf{C}_{k}^{\mathrm{e}}(\omega)+\right.$ $\mathbf{X}_{k}^{\mathrm{e}}(\omega)$ ), which is the upper bound in (4). A simple bisection method can be used to solve the convex optimization problem for calculating the trace-minimal $\omega$.

Prediction Step: In order to model the temporal evolution of the state vector $\underline{\mathrm{x}}_{k}$, a prediction step is carried out by means of the process 
model (6). The predicted estimate is obtained by

$$
\underline{\hat{x}}_{k+1}^{\mathrm{p}}=\mathbf{A}_{k} \underline{x}_{k}^{\mathrm{e}}+\mathbf{B}_{k} \underline{u}_{k} .
$$

The error covariance matrix of the predicted estimate yields

$$
\mathbf{C}_{k+1}^{\mathrm{p}}=\mathbf{A}_{k} \mathbf{C}_{k}^{\mathrm{e}} \mathbf{A}_{k}+\mathbf{C}_{k}^{\mathbf{w}},
$$

and the shape matrix of the set-membership error is given by

$$
\mathbf{X}_{k+1}^{\mathrm{p}}(\omega)=\frac{1}{\omega} \mathbf{A}_{k} \mathbf{X}_{k}^{\mathrm{e}} \mathbf{A}_{k}^{\mathrm{T}}+\frac{1}{1-\omega} \mathbf{X}_{k}^{\mathrm{\omega}},
$$

respectively. In contrast to the filtering step, the parameter $\omega \in(0,1)$ can be determined in closed form in order to minimize the trace, i.e., the bound on the mean squared error.

\subsection{Switching Filtering Scheme}

The generalized Kalman filter has been developed to deal with random and set-bounded error terms simultaneously. For the considered problem of incorporating virtual measurement information into the standard Kalman filtering scheme, the full potential and complexity of the generalized filter does not need to be exploited, and a simplified version can be derived. For physical sensor data, a common stochastic sensor model in the form of (1) is utilized. Setmembership error models like (2) are only utilized for virtual sensor data. This means only special cases of the general sensor model (5) need to be considered.

The standard Kalman filter is initialized with a prior estimate $\underline{\hat{x}}_{0}^{\mathrm{e}}$ with covariance matrix $\mathbf{C}_{0}^{\mathrm{e}}$. For its generalization, also a prior ellipsoid matrix $\mathbf{X}_{0}^{\mathrm{e}}$ has to be defined. However, it can be chosen to be initially zero, i.e., $\mathbf{X}_{0}^{\mathrm{e}}=\mathbf{0}$. In the Kalman filtering scheme, the estimate is dynamically computed in update and prediction steps. In the former step, different formulas for physical and virtual measurements need to be considered. The general structure of the filtering scheme is depicted in Fig. 1. The gain is dependent upon whether actual or virtual sensor evidence is to be incorporated.

Update Step: In order to incorporate physical measurement information $\underline{\hat{z}}_{k}$, the probabilistic model (1) has to be considered. Due to the absence of unknown but bounded measurement noise, the gain (7) can be considerably simplified to

$$
\begin{aligned}
\mathbf{K}_{k}= & \left(\mathbf{X}_{k}^{\mathrm{p}} \mathbf{H}_{k}^{\mathrm{T}}+\mathbf{C}_{k}^{\mathrm{p}} \mathbf{H}_{k}^{\mathrm{T}}\right) . \\
& \left(\mathbf{H}_{k} \mathbf{X}_{k}^{\mathrm{p}} \mathbf{H}_{k}^{\mathrm{T}}+\mathbf{H}_{k} \mathbf{C}_{k}^{\mathrm{p}} \mathbf{H}^{\mathrm{T}}+\mathbf{C}_{k}^{\mathbf{v}}\right)^{-1} .
\end{aligned}
$$

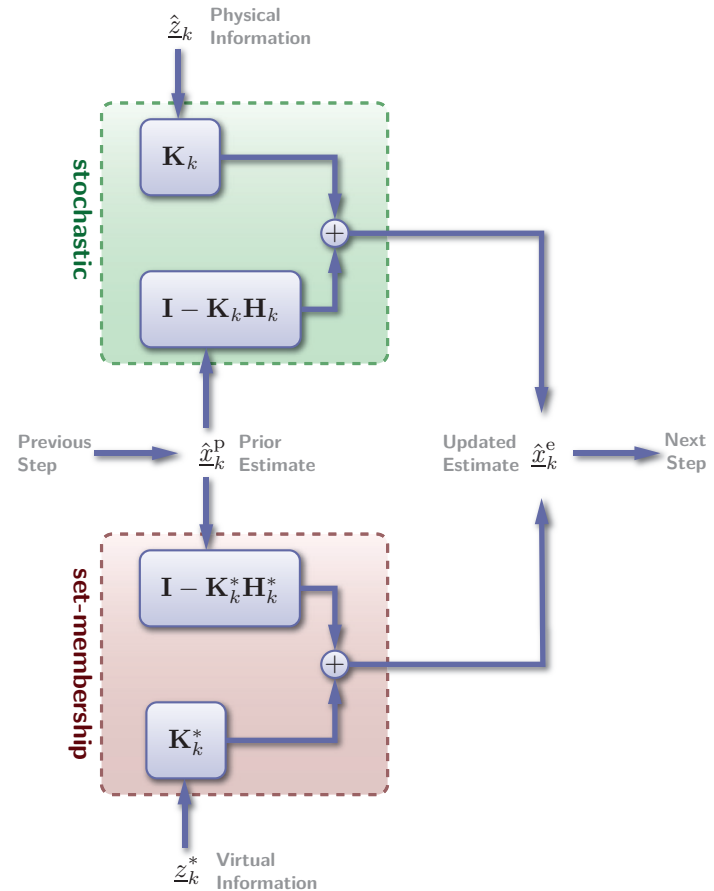

Fig. 1: Structure of filtering scheme with physical and virtual sensor evidence.

The update formulas (8) for the estimate and the covariance matrix become

$$
\underline{\hat{x}}_{k}^{\mathrm{e}}=\left(\mathbf{I}-\mathbf{K}_{k} \mathbf{H}_{k}\right) \underline{\hat{x}}_{k}^{\mathrm{p}}+\mathbf{K}_{k} \underline{\hat{z}}_{k}
$$

and

$$
\begin{aligned}
\mathbf{C}_{k}^{\mathrm{e}}= & \left(\mathbf{I}-\mathbf{K}_{k} \mathbf{H}_{k}\right) \mathbf{C}_{k}^{\mathrm{p}}\left(\mathbf{I}-\mathbf{K}_{k} \mathbf{H}_{k}\right)^{\mathrm{T}} \\
& +\mathbf{K}_{k} \mathbf{C}_{k}^{\mathbf{v}} \mathbf{K}_{k}^{\mathrm{T}},
\end{aligned}
$$

and, in particular, the ellipsoidal shape matrix reduces to

$$
\mathbf{X}_{k}^{\mathrm{e}}=\left(\mathbf{I}-\mathbf{K}_{k} \mathbf{H}_{k}\right) \mathbf{X}_{k}^{\mathrm{p}}\left(\mathbf{I}-\mathbf{K}_{k} \mathbf{H}_{k}\right)^{\mathrm{T}} .
$$

Apparently, the update with physical measurement information does not require a minimization over the parameter $\omega$.

For virtual measurement information that can be represented as a bounded set, the model (2) is utilized. The required gain, in this case, becomes

$$
\begin{aligned}
& \mathbf{K}_{k}^{*}(\omega)=\left(\frac{1}{\omega} \mathbf{X}_{k}^{\mathrm{p}}\left(\mathbf{H}_{k}^{*}\right)^{\mathrm{T}}+\mathbf{C}_{k}^{\mathrm{p}}\left(\mathbf{H}_{k}^{*}\right)^{\mathrm{T}}\right) . \\
& \left(\frac{1}{\omega} \mathbf{H}_{k}^{*} \mathbf{X}_{k}^{\mathrm{p}}\left(\mathbf{H}_{k}^{*}\right)^{\mathrm{T}}+\frac{1}{1-\omega} \mathbf{X}_{k}^{*}+\mathbf{H}_{k}^{*} \mathbf{C}_{k}^{\mathrm{p}}\left(\mathbf{H}^{*}\right)^{\mathrm{T}}\right)^{-1},
\end{aligned}
$$

which is due to the vanishing covariance matrix $\mathbf{C}_{k}^{\mathbf{v}}=\mathbf{0}$. This gain is is employed to compute the updated mean

$$
\underline{\hat{\gamma}}_{k}^{\mathrm{e}}(\omega)=\left(\mathbf{I}-\mathbf{K}_{k}^{*}(\omega) \mathbf{H}_{k}\right) \underline{\hat{x}}_{k}^{\mathrm{p}}+\mathbf{K}_{k}^{*}(\omega) \underline{z}_{k}^{*},
$$


the updated covariance matrix

$$
\mathbf{C}_{k}^{\mathrm{e}}(\omega)=\left(\mathbf{I}-\mathbf{K}_{k}^{*}(\omega) \mathbf{H}_{k}^{*}\right) \mathbf{C}_{k}^{\mathrm{p}}\left(\mathbf{I}-\mathbf{K}_{k}^{*}(\omega) \mathbf{H}_{k}^{*}\right)^{\mathrm{T}},
$$

and the updated ellipsoid shape matrix

$$
\begin{aligned}
\mathbf{X}_{k}^{\mathrm{e}}(\omega)= & \frac{1}{\omega}\left(\mathbf{I}-\mathbf{K}_{k}^{*}(\omega) \mathbf{H}_{k}\right) \mathbf{X}_{k}^{\mathrm{p}}\left(\mathbf{I}-\mathbf{K}_{k}^{*}(\omega) \mathbf{H}_{k}\right)^{\mathrm{T}} \\
& +\frac{1}{1-\omega} \mathbf{K}_{k}^{*}(\omega) \mathbf{X}_{k}^{*} \mathbf{K}_{k}^{*}(\omega)^{\mathrm{T}} .
\end{aligned}
$$

As stated before, the parameter $\omega$ is to be chosen from the interval $(0,1)$. Consequently, in order to obtain a trace-minimal result, a numerical optimization has to be performed. In contrast to the previous subsection, this procedure is only required if virtual information is to be incorporated.

Prediction Step: In many applications, discrete-time models are derived from stochastic processes. The prediction step of the standard Kalman filter relies on a purely stochastic system model

$$
\underline{\mathbf{x}}_{k+1}=\mathbf{A}_{k} \underline{\mathbf{x}}_{k}+\mathbf{B}_{k} \underline{u}_{k}+\underline{\mathbf{w}}_{k}
$$

in order to compute the current state estimate according to

$$
\underline{\hat{x}}_{k+1}^{\mathrm{p}}=\mathbf{A}_{k} \underline{\hat{x}}_{k}^{\mathrm{e}}+\mathbf{B}_{k} \underline{u}_{k}
$$

and

$$
\mathbf{C}_{k+1}^{\mathrm{p}}=\mathbf{A}_{k} \mathbf{C}_{k}^{\mathrm{e}} \mathbf{A}_{k}^{\mathrm{T}}+\mathbf{C}_{k}^{\mathrm{w}}
$$

for the conditional mean and the error covariance matrix, respectively. The shape matrix of the ellipsoidal error bound is propagated in time by

$$
\mathbf{X}_{k+1}^{\mathrm{p}}=\mathbf{A}_{k} \mathbf{X}_{k}^{\mathrm{e}} \mathbf{A}_{k}^{\mathrm{T}},
$$

and no weighting parameter needs to be determined. Hence, in applications where purely stochastic process models are considered, the calculation of $\mathbf{X}_{k+1}^{\mathrm{p}}$ reduces to the simple transformation (10c). The formulas (10a) and (10b) for the mean and covariance matrix still correspond to the standard Kalman filter.

\subsection{Discussion on Applications}

The switching filtering scheme allows easily incorporating virtual measurement information that can be modeled as an ellipsoidal set. Virtual measurement information that can still be represented by normally distributed random variables can be treated by means of the standard Kalman filter. However, many examples can be identified where set-membership representations are particularly well suited to treat virtual information. The following examples shall elucidate the use of set-membership models for virtual measurements.
State constraints are, for example, bounds on acceleration values of a mobile robot. In case of a near-constant-acceleration model [13] for target tracking in a plane, the state consists of six components, of which two components $\underline{a}=\left[a_{x_{1}}, a_{x_{2}}\right]^{\mathrm{T}}$ refer to the acceleration values in each direction. An acceleration constraint can be modeled as a bound on the norm $\|\underline{a}\|_{2}=\left\|\mathbf{H}_{a} \underline{x}\right\|_{2} \leq b$, where $\mathbf{H}_{a}$ selects the acceleration vector of the state $\underline{x}$ and which is equivalent to the ellipsoidal constraint

$$
\frac{1}{b^{2}}\|\underline{a}\|_{2}^{2}=\left(\mathbf{H}_{a} \underline{x}\right)^{\mathrm{T}} \mathbf{X}_{a}^{-1}\left(\mathbf{H}_{a} \underline{x}\right) \leq 1
$$

with $\mathbf{X}_{a}=b^{2} \mathbf{I} \in \mathbb{R}^{2 \times 2}$, i.e., $\underline{a}=\mathbf{H}_{a} \underline{x} \in \mathcal{E}\left(\underline{0}, \mathbf{X}_{a}\right)$. Hence, this constraint can be reformulated as a set-bounded measurement of the acceleration components.

Environmental constraints relate to external conditions to which the state is constrained. For instance, the width of a street can be regarded as a constraint on the position of a road vehicle to be tracked [14]. This constraint can be represented by an interval, which is orthogonal to the direction of the road, i.e., a straight road segment can be modeled with the aid the Hessian normal form

$$
\underline{n}^{\mathrm{T}} \cdot\left(\mathbf{H}_{p} \underline{x}\right)=d,
$$

where $\underline{n}$ is the unit normal vector being orthogonal to the direction of the road segment and $d$ is its distance to the origin. The matrix $\mathbf{H}_{p}$ maps the state $\underline{x}$ to the components comprising the position. Using the road width $w$ as a constraint corresponds to the interval $\left|\underline{n}^{\mathrm{T}} \cdot\left(\mathbf{H}_{p} \underline{x}\right)-d\right| \leq w$, which can directly be reformulated into an onedimensional ellipsoid

$$
\left(\mathbf{H}_{s} \underline{x}-d\right)^{\mathrm{T}} \mathbf{X}_{s}^{-1}\left(\mathbf{H}_{s} \underline{x}-d\right) \leq 1
$$

with $\mathbf{X}_{s}=w^{2}$ and $\mathbf{H}_{s}=\underline{n}^{\mathrm{T}} \mathbf{H}_{p}$. This constraint complies with the virtual measurement information $\mathbf{H}_{s} \underline{x} \in \mathcal{E}\left(d, \mathbf{X}_{s}\right)$.

Missing observations can often be translated into virtual measurement information if certain triggering thresholds are known and can be exploited. For instance in case of a ground moving target indicator radar, a threshold with respect to the radial velocity of the target to be tracked needs to be reached in order to detect the target [6]. As a simple model, the threshold can be defined with respect to the normalized direction of the line of sight $\underline{n}_{\text {los }}$, and measurements can be obtained if the velocity vector $\underline{v}$ projected onto $\underline{n}_{\text {los }}$ exceeds the threshold $t$, i.e., $\underline{n}_{\text {los }}^{\mathrm{T}}>t$. Reversely, missing observations can 
be translated into virtual sensor evidence. In this case, the condition $\underline{n}_{\operatorname{los}}^{\mathrm{T}} \underline{v} \leq t$ can be translated into the ellipsoidal constraint

$$
\left(\mathbf{H}_{n} \underline{x}\right)^{\mathrm{T}} \mathbf{X}_{n}^{-1}\left(\mathbf{H}_{n} \underline{x}\right) \leq 1
$$

with $\mathbf{X}_{v}=t^{2}$ and $\mathbf{H}_{n}=\underline{n}_{\mathrm{los}} \mathbf{H}_{v}$, where the matrix $\mathbf{H}_{v}$ selects the velocity components of the state $\underline{x}$. In line with (2a), the ellipsoidal measurement $\mathbf{H}_{n} \underline{x} \in \mathcal{E}\left(0, \mathbf{X}_{v}\right)$ can be defined.

\section{Conclusions}

Virtual measurements have been defined as evidence that can be deduced from additional background knowledge about the considered phenomenon. In order to take advantage of this type of information, specific measurement models need to be derived. Different strategies can be pursued in order to model and incorporate virtual measurement information. In this work, a simple set-membership characterization of virtual information has been proposed that can easily be integrated into the prediction-correction cycle of the Kalman filter. The proposed technique encompasses an alternative to a purely stochastic approach, which relies on the definition of specific likelihood functions. Such a stochastic approach often suffers from the problem that non-Gaussian density functions and nonlinear models need to be considered. By contrast, if a set-membership and, in particular, ellipsoidal representation of virtual information can be attained, an easyto-implement generalization of the Kalman filter can be exploited. The attractiveness of the proposed technique mainly lies in its simplicity. In the filtering step, the algorithm switches between a stochastic sensor model for actual sensor data and an ellipsoidal measurement model for virtual information. Only the latter update step requires the numerical computation of a bounded scalar weighting parameter while the former update step as well as the prediction step correspond to the standard Kalman filter. Consequently, the incorporation of virtual measurement information has been boiled down to a simple measurement update with an ellipsoidal error representation.

\section{References}

[1] D. Simon, "Kalman Filtering with State Constraints: A Survey of Linear and Nonlinear Algorithms," IET Control Theory \& Applications, vol. 4, no. 8, pp. 1303-1318, Aug. 2010.

[2] B. J. Spivey, J. D. Hedengren, and T. F. Edgar, "Constrained Nonlinear Estimation for Industrial Process Fouling," Industrial \& Engineering Chemistry Research, vol. 49, no. 17, pp. 78247831, 2010.
[3] C. Yang and E. Blasch, "Fusion of Tracks with Road Constraints," Journal of Advances in Information Fusion, vol. 3, no. 1, pp. 14-32, Jun. 2008.

[4] S. Thrun, W. Burgard, and D. Fox, Probabilistic Robotics, ser. Intelligent Robotics and Autonomous Agents. MIT Press, 2005.

[5] J. Sijs, B. Noack, and U. D. Hanebeck, "Eventbased State Estimation with Negative Information," in Proceedings of the 16th International Conference on Information Fusion (Fusion 2013), Istanbul, Turkey, Jul. 2013.

[6] C. S. Agate, R. M. Wilkerson, and K. J. Sullivan, "Utilizing Negative Information to Track Ground Vehicles through Move-Stop-Move Cycles," in Proceedings of SPIE - Signal Processing, Sensor Fusion, and Target Recognition XIII, vol. 5429, Orlando, Florida, USA, Apr. 2004.

[7] W. Koch, "On Exploiting 'Negative" Sensor Evidence for Target Tracking and Sensor Data Fusion," Information Fusion, vol. 8, no. 1, pp. 2839, Jan. 2005.

[8] S. Jovanoska, F. Govaers, R. Thomä, and W. Koch, "Dynamic-occlusion Likelihood Incorporation in a PHD Filter Based Range-only Tracking System," in Proceedings of the 18th International Conference on Information Fusion (Fusion 2015), Whashington, D.C., USA, Jul. 2015.

[9] J. Hoffmann, M. Spranger, D. Göhring, and M. Jüngel, "Making Use Of What You Don't See: Negative Information In Markov Localization," in Proceedings of the 2005 IEEE/RSJ International Conference on Intelligent Robots and Systems (IROS 2005), Edmonton, Canada, Aug. 2005.

[10] K. Tischler and H. S. Vogt, "A Sensor Data Fusion Approach for the Integration of Negative Information," in Proceedings of the 10th International Conference on Information Fusion (Fusion 2007), Quebec, Canada, Jul. 2007.

[11] B. Noack, F. Pfaff, and U. D. Hanebeck, "Optimal Kalman Gains for Combined Stochastic and Set-Membership State Estimation," in Proceedings of the 51st IEEE Conference on Decision and Control (CDC 2012), Maui, Hawaii, USA, Dec. 2012.

[12] B. Noack, State Estimation for Distributed Systems with Stochastic and Set-membership Uncertainties, ser. Karlsruhe Series on Intelligent Sensor-Actuator-Systems $14 . \quad$ Karlsruhe, Germany: KIT Scientific Publishing, 2013.

[13] X.-R. Li and V. P. Jilkov, "Survey of Maneuvering Target Tracking III. Measurement Models," in Proceedings of SPIE - Signal and Data Processing of Small Targets 2001, San Diego, California, USA, Jul. 2001.

[14] B. Noack, M. Baum, and U. D. Hanebeck, "State Estimation for Ellipsoidally Constrained Dynamic Systems with Set-membership Pseudo Measurements," in Proceedings of the 2015 IEEE International Conference on Multisensor Fusion and Integration for Intelligent Systems (MFI 2015), San Diego, California, USA, Sep. 2015. 